\title{
Letters
}

\section{Reply from Dr L Waller}

Before replying to the questions, I would like once more to emphasise that our request for information before the study was neglected and that the clinical part of the study has been reproducible ever since the initial presentation at the regional meeting of IMSOP in Cyprus in April 1995.

1 It has proved suitable to use the dynamometer the way it was used in the study. Our nurse was informed about the importance of keeping strictly to the same technique in all measurements and trained for some time to standardise her methodology before the actual study started. The velocity was not recorded due to practical reasons. Although there may have been small differences in angles, velocity and acceleration, these can be ignored in view of the large number of measurements $(n=526)$. The statistical analysis also pointed to a significant difference between the two catheters $(p<0.001)$.

2 In clinical trials adverse events (AE) must be reported. In the study two reporting methods were used. One of them consisted of the AE that occurred during the measurements and reported by the nurse performing them, while the other comprised AE reported by patients and the rest of the staff. It is important to inform about the AE frequency in a study so we present those reported by the nurse. The relationship between sticking and dynomometer value can be seen in the article. It should also be mentioned that the study was designed to use tap water as lubricator but due to severe stickings when using the EasiCath we had to change to saline solution.

3 Measurement of the osmolality of the urethral epithelium has proved to be difficult. The actual sampling of the cell layer destroys the cells, which makes measurement of osmolality impossible. However, this is not inconsistent with the logic of our theory, namely that the outer layer of a hydrophilic catheter should equalise what normally passes through the urethra.

According to the original abstract (your ref. 1), the osmolality of human urine is $700-1100 \mathrm{mOsm} / \mathrm{kg}$. The absence of a relationship between friction and osmolality was, in our view, due to the short catheterisation time $(60 \mathrm{~s})$. In the present study the catheterisation time was slightly more than 4 min.

4 The results of our first study (your ref. 1) show a clear relationship between friction and trauma of the urethral epithelium. In this study there was a clear relationship between removal friction and the osmolality of the outer layer of hydrophilic catheters. Therefore, we assume that there is also a relationship between trauma and osmolality, although it remains for this to be demonstrated scientifically. We have now experienced on two occasions severe stickings and stuck catheters when using lowosmolar catheters and so have a very good reason for assuming the existence of such a relationship.

5 The aim of this study was to ascertain the significance of the osmolality of the outer layer of hydrophilic catheters. According to the laboratory tests performed, both the catheters were PVC catheters with a hydrophilic coating, while LoFric $^{\mathbb{R}}$ also had an outer layer of sodium chloride. No other significant differences was observed between the two catheters.

L Waller, Eklandagatan 52,C 41261 Göteborg. Sweden 